\title{
Gravitational-darkening of Altair from interferometry
}

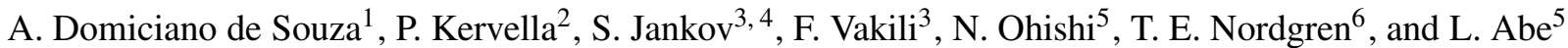 \\ 1 Max-Planck-Institut für Radioastronomie, Auf dem Hügel 69, 53121 Bonn, Germany \\ e-mail: adomicia@mpifr-bonn.mpg.de \\ 2 LESIA, UMR 8109, Observatoire de Paris-Meudon, 5 place Jules Janssen, 92195 Meudon Cedex, France \\ 3 Lab. Univ. d'Astrophysique de Nice (LUAN), UMR 6525, UNSA, Parc Valrose, 06108 Nice Cedex 02, France \\ 4 Astronomical Observatory Belgrade, MNTRS 1940, Volgina 7, 11050 Beograd, Serbia and Montenegro \\ 5 National Astronomical Observatory of Japan, 2-21-1 Osawa, Mitaka, Tokyo 181-8588, Japan \\ ${ }^{6}$ Department of Physics, University of Redlands, 1200 East Colton Avenue, Redlands, CA 92373, USA
}

Received 3 December 2004 / Accepted 13 June 2005

\section{ABSTRACT}

Interferometric observations have revealed that the rapid rotator Altair is a flattened star with a non-centrally symmetric intensity distribution. In this work we perform for the first time a physically consistent analysis of all interferometric data available so far, corresponding to three different interferometers operating in several spectral bands. These observations include new data (squared visibilities in the $H$ and $K$ bands from VLTI-VINCI) as well as previously published data (squared visibilities in the $K$ band from PTI and squared visibilities, triple amplitudes, and closure phases in the visible between $520 \mathrm{~nm}$ and $850 \mathrm{~nm}$ from NPOI). To analyze these data we perform a $\chi^{2}$ minimization using an interferometry-oriented model for fast rotators, which includes Roche approximation, limb-darkening, and von Zeipel-like gravity-darkening. Thanks to the rich interferometric data set available and to this physical model, the main uniqueness problems were avoided. As a result, we show that the observations can only be explained if Altair has a gravity-darkening compatible with the expected value for hot stars, i.e., the von Zeipel effect $\left(T_{\text {eff }} \propto g^{0.25}\right)$.

Key words. techniques: high angular resolution - techniques: interferometric - methods: data analysis - stars: rotation stars: individual: Altair

\section{Introduction}

Altair ( $\alpha$ Aql, HR 7557, HD 187642) is a bright $(V=0.77)$, rapidly rotating A7IV-V star, which has been studied by many authors. For example, Buzasi et al. (2005) recently detected several oscillating frequencies in Altair and proved that this star is a variable of the $\delta$ Scuti type, as expected by its location within the instability strip. Several basic physical parameters of Altair are summarized by Buzasi et al. (2005) in their introduction. One important characteristic of Altair is its fast rotation. Spectroscopic and interferometric observations indicate a $v_{\text {eq }} \sin i$ value between $190 \mathrm{~km} \mathrm{~s}^{-1}$ and $250 \mathrm{~km} \mathrm{~s}^{-1}$ (Abt \& Morrell 1995; van Belle et al. 2001; Royer et al. 2002; among others); most recently Reiners \& Royer (2004) determined $v_{\text {eq }} \sin i=227 \pm 11 \mathrm{~km} \mathrm{~s}^{-1}$ from spectroscopy.

Theories foresee that such a high rotation velocity can lead to many modifications in the physical structure of a star like Altair. In particular, the star is expected (1) to be oblate because of a strong centrifugal force and (2) to exhibit gravity-darkening (after the seminal work of von Zeipel 1924). These theoretically expected modifications are now measured by modern observing techniques, notably those based on long baseline interferometry. See, for example,
Domiciano de Souza et al. (2003) for the case of another rapidly rotating star (Achernar).

For Altair, van Belle et al. (2001) measured the rotational flattening projected onto the sky-plane using the Palomar Testbed Interferometer (PTI, Colavita et al. 1999). By adopting an equivalent limb-darkened ellipse model these authors derived major and minor axes of $2 a=3.461 \pm 0.038$ mas and $2 b=3.037 \pm 0.069$ mas, respectively, which means an axial ratio of $a / b=1.140 \pm 0.029$. van Belle et al. (2001) used a Roche model (solid body rotation and mass $M$ concentrated in a point at the center of the star) without gravity-darkening to analyze their observations of Altair. However, even with this relatively simplified model their analysis encountered several uniqueness problems caused by the limited coverage of spatial frequencies (observations inside the first visibility lobe only) and spectral information (only one broadband near-IR filter used). The important issue of uniqueness problems in interferometric studies of rapidly rotating stars is discussed by Domiciano de Souza et al. (2002).

More recently, Ohishi et al. (2004) used the Navy Prototype Optical Interferometer (NPOI, Armstrong et al. 1998) to observe Altair. In particular, they obtained closure phases and 
squared visibilities around the first minimum. These observations suggest that Altair is not only oblate but also that it is a gravity-darkened star with a non-centrally symmetric intensity distribution. The nature of these data largely diminishes the uniqueness problems associated with the analysis of rapid rotators.

Even though the observations indicate that Altair is an oblate and gravity-darkened star, previous works did not adopt physically consistent models including these two effects. In the present work we use our interferometry-oriented model for fast rotators (Domiciano de Souza et al. 2002) to perform a $\chi^{2}$ minimization including all interferometric data available up-to-date: new squared visibilities in the $H$ and $K$ bands from the Very Large Telescope Interferometer (VLTI, e.g., Glindemann et al. 2003); (2) squared visibilities in the $K$ band from PTI; and (3) squared visibilities, triple amplitudes, and closure phases in the visible from NPOI.

In Sect. 2 we summarize the observations used here, and in Sect. 3 we describe the adopted model, which includes Roche approximation, a limb-darkening law compatible with Altair's effective temperature distribution, and a von Zeipel-like gravity-darkening law. In Sect. 4 we present the main results of our $\chi^{2}$ analysis of the interferometric data. A critical discussion of our results is given in Sect. 5, while the conclusions of this work are summarized in Sect. 6 .

\section{Interferometric observations}

The first attempt to measure the geometrical deformation of Altair was carried out with the Narrabri intensity interferometer (Hanbury Brown 1974). However, these observations remained too marginal to allow unambiguous conclusions to be drawn by the Australian group. Recent observations by at least three modern interferometers have resulted in several high-quality measurements becoming available. In the following section we briefly describe the three interferometric data sets (one new and two previously published and analyzed) used in this work to constrain a number of unknown physical parameters of Altair.

\subsection{VLTI-VINCI near-IR observations}

We first describe the new near-IR observations of Altair performed with the VLTI. These new data were obtained with two test siderostats $(0.35 \mathrm{~m}$ aperture $)$ and the VINCI ${ }^{1}$ instrument (Kervella et al. 2000 and Kervella et al. 2003a). The visibility measurements were all recorded on the E0-G1 baseline of the VLTI (ground length of $66 \mathrm{~m}$ ). We combined the stellar light using a classical fiber-based triple coupler (MONA) for the $K$ band observations, and an integrated optics beam combiner (IONIC, Lebouquin et al. 2004) in the $H$ band. Standard $K(2.0<\lambda<2.4 \mu \mathrm{m})$ and $H(1.5<\lambda<1.8 \mu \mathrm{m})$ band filters were used for these observations. The effective wavelength of the observations changes slightly depending on the spectral type of the observed target. For Altair, we determined $\lambda_{\text {eff }}=2.176 \pm 0.003 \mu \mathrm{m}$ and $\lambda_{\text {eff }}=1.633 \pm 0.003 \mu \mathrm{m}$, respectively in the $K$ and $H$ bands.

\footnotetext{
1 V(LT) IN(terferometer) C(ommissioning) I(nstrument).
}

The raw data processing has been achieved using a waveletbased algorithm, integrated in an automated data reduction pipeline (Kervella et al. 2004a). The general principle is similar to the original FLUOR algorithm (Coudé du Foresto et al. 1997), but instead of the classical Fourier analysis, we implemented a wavelet-based time-frequency analysis (Ségransan et al. 1999). The two calibrated output interferograms are subtracted to correct for residual photometric fluctuations. The output of the pipeline is a single value of the squared coherence factor $\mu^{2}$ for each series of 500 interferograms and the associated bootstrapped error bar. We obtained a total of 5500 interferograms of Altair in the $K$ band and 4500 in the $H$ band, among which 2749 and 1949 were reduced by the pipeline, respectively. All the VINCI data were obtained between July and September 2002. The final normalized squared visibilities $V^{2}$ and other observational information are given in Table 1.

We used three stars as calibrators for the $K$ band observations (24Cap, $\chi$ Phe, and $70 \mathrm{Aql}$ ) and one for the $H$ band ( $\alpha$ Ind). These stars were selected from Cohen et al. (1999) for their stability, and we took their sizes from the Bordé et al. (2002) catalogue. To obtain their equivalent uniform-disk diameters, we applied the broadband limb-darkening corrections provided by Claret (2000a), based on the ATLAS models (Kurucz 1992). In absence of data in the literature, the metallicity of the calibration stars was taken as solar. Note, however, that in the $H$ and $K$ bands the influence of metallicity on the limb-darkening is very weak. The relevant properties of the calibrators used for VINCI observations are listed in Table 2.

The choice of the calibrators is an important step for preparing interferometric observations, since significant departures of their actual visibilities from the expected model can propagate into biases on the calibrated visibilities of the scientific target. Among the possible reasons for such departures, binarity (or multiplicity in general) and deviations from spherical symmetry (due, for instance, to fast rotation or gravitational interaction) are the most critical. All stars in the Cohen et al. (1999) catalogue were carefully scrutinized by these authors for the presence of companions and are currently regarded as single stars. With respect to fast rotation, the value of $v_{\mathrm{eq}} \sin i$ is only available for one star, $70 \mathrm{Aql}$, which is also the most sensitive star to potential deformations as it is a bright giant. With $v_{\text {eq }} \sin i=1.9 \mathrm{~km} \mathrm{~s}^{-1}, \log g \approx 1.9$, and a radius of $\approx 200 R_{\odot}$, a first order approximation of its flattening ratio (Roche model) is given by the following relation (e.g. Domiciano de Souza et al. 2002):

$\frac{R_{\mathrm{eq}}}{R_{\mathrm{p}}}=1+\frac{v_{\mathrm{eq}}^{2} R_{\mathrm{eq}}}{2 G M}<1+10^{-4}$

The effect of the rotation on the shape of this star is therefore taken as negligible. Measurements of the projected rotational velocities are not available for the other calibrators of our sample, but as they are giant stars, we assume that they are small enough so that their rotational deformation can be neglected. Our four calibrators are significantly resolved by the interferometer, but the a priori uncertainty on their angular diameters was carefully propagated to the final error bars on the calibrated squared visibilities of Altair. The errors in the calibrated $V^{2}$ 
Table 1. VLTI-VINCI observations of Altair performed in the $H$ and $K$ bands.

\begin{tabular}{|c|c|c|c|c|c|c|c|c|}
\hline$H$ band & & & & & & & & \\
\hline $\begin{array}{l}\text { Date } \\
\text { (JD) }\end{array}$ & $\begin{array}{c}\text { Projected } \\
\text { Baseline }(\mathrm{m})\end{array}$ & $\begin{array}{c}\text { Position } \\
\text { Angle }^{a} \text { (deg) }\end{array}$ & $\begin{array}{c}\text { Calib. } \\
V^{2}\end{array}$ & $\begin{array}{c}\text { Stat. } \\
V^{2} \text { error }\end{array}$ & $\begin{array}{c}\text { Syst. } \\
V^{2} \text { error }\end{array}$ & $\begin{array}{c}\text { Total } \\
V^{2} \text { error }\end{array}$ & $\begin{array}{c}\text { Uniform disc } \\
\text { diameter (mas) }\end{array}$ & Calibrator \\
\hline 2452477.655 & 62.110 & 139.77 & 0.388 & \pm 0.022 & \pm 0.008 & \pm 0.023 & $3.22 \pm 0.09$ & $\alpha$ Ind \\
\hline 2452477.659 & 61.702 & 139.97 & 0.403 & \pm 0.023 & \pm 0.009 & \pm 0.024 & $3.18 \pm 0.10$ & $\alpha$ Ind \\
\hline 2452479.561 & 65.950 & 140.66 & 0.336 & \pm 0.023 & \pm 0.006 & \pm 0.023 & $3.23 \pm 0.09$ & $\alpha$ Ind \\
\hline 2452479.706 & 55.760 & 144.48 & 0.464 & \pm 0.027 & \pm 0.009 & \pm 0.028 & $3.26 \pm 0.12$ & $\alpha$ Ind \\
\hline 2452482.726 & 52.131 & 148.77 & 0.510 & \pm 0.052 & \pm 0.012 & \pm 0.054 & $3.28 \pm 0.24$ & $\alpha$ Ind \\
\hline 2452483.645 & 61.490 & 140.08 & 0.401 & \pm 0.024 & \pm 0.006 & \pm 0.024 & $3.20 \pm 0.10$ & $\alpha$ Ind \\
\hline 2452484.699 & 54.905 & 145.37 & 0.539 & \pm 0.042 & \pm 0.010 & \pm 0.043 & $2.99 \pm 0.18$ & $\alpha$ Ind \\
\hline 2452485.594 & 64.851 & 139.08 & 0.371 & \pm 0.032 & \pm 0.008 & \pm 0.033 & $3.15 \pm 0.13$ & $\alpha$ Ind \\
\hline 2452485.598 & 64.627 & 139.07 & 0.358 & \pm 0.032 & \pm 0.008 & \pm 0.033 & $3.21 \pm 0.13$ & $\alpha$ Ind \\
\hline \multicolumn{9}{|l|}{$K$ band } \\
\hline $\begin{array}{l}\text { Date } \\
\text { (JD) }\end{array}$ & $\begin{array}{c}\text { Projected } \\
\text { Baseline }(\mathrm{m})\end{array}$ & $\begin{array}{c}\text { Position } \\
\text { Angle (deg) }\end{array}$ & $\begin{array}{c}\text { Calib. } \\
V^{2}\end{array}$ & $\begin{array}{c}\text { Stat. } \\
V^{2} \text { error }\end{array}$ & $\begin{array}{c}\text { Syst. } \\
V^{2} \text { error }\end{array}$ & $\begin{array}{c}\text { Total } \\
V^{2} \text { error }\end{array}$ & $\begin{array}{c}\text { Uniform disc } \\
\text { diameter (mas) }\end{array}$ & Calibrator \\
\hline 2452469.722 & 57.285 & 143.05 & 0.656 & \pm 0.017 & \pm 0.016 & \pm 0.023 & $3.18 \pm 0.13$ & 24 Cap \\
\hline 2452469.755 & 53.065 & 147.53 & 0.685 & \pm 0.015 & \pm 0.017 & \pm 0.023 & $3.26 \pm 0.14$ & 24 Cap \\
\hline 2452469.763 & 51.957 & 149.02 & 0.696 & \pm 0.017 & \pm 0.017 & \pm 0.024 & $3.26 \pm 0.15$ & 24 Cap \\
\hline 2452531.587 & 52.790 & 147.89 & 0.645 & \pm 0.072 & \pm 0.007 & \pm 0.072 & $3.52 \pm 0.44$ & $\chi$ Phe \\
\hline 2452531.592 & 52.204 & 148.67 & 0.632 & \pm 0.076 & \pm 0.007 & \pm 0.076 & $3.64 \pm 0.47$ & $\chi$ Phe \\
\hline 2452531.596 & 51.624 & 149.49 & 0.663 & \pm 0.076 & \pm 0.007 & \pm 0.076 & $3.49 \pm 0.48$ & $\chi$ Phe \\
\hline 2452536.511 & 60.454 & 140.68 & 0.605 & \pm 0.028 & \pm 0.008 & \pm 0.029 & $3.28 \pm 0.15$ & $70 \mathrm{Aql}$ \\
\hline 2452536.543 & 56.759 & 143.52 & 0.636 & \pm 0.031 & \pm 0.008 & \pm 0.032 & $3.32 \pm 0.18$ & $70 \mathrm{Aql}$ \\
\hline 2452536.547 & 56.226 & 144.03 & 0.701 & \pm 0.033 & \pm 0.008 & \pm 0.034 & $2.98 \pm 0.20$ & $70 \mathrm{Aql}$ \\
\hline 2452536.578 & 52.212 & 148.66 & 0.713 & \pm 0.049 & \pm 0.009 & \pm 0.050 & $3.14 \pm 0.33$ & $70 \mathrm{Aql}$ \\
\hline 2452536.582 & 51.738 & 149.33 & 0.643 & \pm 0.043 & \pm 0.008 & \pm 0.043 & $3.60 \pm 0.27$ & $70 \mathrm{Aql}$ \\
\hline
\end{tabular}

${ }^{a} 0^{\circ}$ is North and $90^{\circ}$ is East.

Table 2. Relevant parameters of the calibrators used for VINCI observations of Altair.

\begin{tabular}{lcccc}
\hline \hline Name & 24 Cap & $\chi$ Phe & 70 Aql & $\alpha$ Ind \\
HD number & HD 200914 & HD 12524 & HD 196321 & HD 196171 \\
\hline$m_{\mathrm{V}}$ & 4.5 & 5.2 & 4.9 & 3.1 \\
$m_{\mathrm{K}}$ & 0.5 & 1.3 & 1.2 & 0.9 \\
Sp. type & $\mathrm{M} 0.5 \mathrm{III}$ & K5III & K5II & K0III \\
$T_{\text {eff }}(\mathrm{K})^{a}$ & 3630 & 3780 & 3780 & 4720 \\
$\log g^{\mathrm{a}}$ & 1.4 & 1.9 & 1.9 & 2.6 \\
{$[\mathrm{Fe} / \mathrm{H}]^{c}$} & - & - & - & 0.0 \\
$v_{\text {eq }} \sin i\left(\mathrm{~km} \mathrm{~s}^{-1}\right)^{d}$ & - & - & 1.9 & - \\
$\oslash_{\mathrm{LD}}(\mathrm{mas})^{a}$ & $4.43 \pm 0.05$ & $2.77 \pm 0.03$ & $3.27 \pm 0.04$ & $3.28 \pm 0.03$ \\
$\oslash_{\mathrm{UD}}(\mathrm{mas})^{b}$ & $4.30 \pm 0.05$ & $2.69 \pm 0.03$ & $3.17 \pm 0.04$ & $3.20 \pm 0.03$ \\
\hline
\end{tabular}

a From Cohen et al. (1999).

${ }^{b}$ Limb-darkened disc diameters $\oslash_{\mathrm{LD}}$ converted to uniform disc diameters $\oslash_{\mathrm{UD}}$ using the linear limb-darkening coefficients from Claret (2000a).

c From Cayrel de Strobel et al. (1997, 2001).

$d$ From Glebocki et al. (2000).

(statistical, systematic, and total) are also listed in Table 1. The uncertainties in $V^{2}$ are dominated by the statistical errors.

\subsection{PTI near-IR observations}

Another data set used in this paper was obtained with PTI and was previously reported and analyzed by van Belle et al. (2001, hereafter vB2001). This data set corresponds to
27 measurements of $V^{2}$ on Altair performed in the $K$ band for two distinct baselines (ground lengths of $85 \mathrm{~m}$ and $110 \mathrm{~m}$ ).

\subsection{NPOI visible observations}

The third data set used in this work, which was previously reported and analyzed by Ohishi et al. (2004, hereafter ONH2004), corresponds to interferometric observations in the 
visible obtained with NPOI. These observations of Altair were recorded simultaneously using three baselines forming a triangle (ground lengths of 30,37, and $64 \mathrm{~m}$ ), allowing measurements of $V^{2}$, triple amplitudes, and closure phases. In this work we use the NPOI observations of Altair performed on May 252001 (see Table 2 of ONH2004), which consist of 7 scans (Hummel et al. 1998). We use 18 spectral channels covering wavelengths from 520 to $850 \mathrm{~nm}$; the data for $\lambda=633 \mathrm{~nm}$ are not used because they contain light from the metrology laser, and the data for $\lambda=618 \mathrm{~nm}$ are not used because they are not available for all NPOI observables.

\section{Modeling Altair}

\subsection{Model of a rotating star}

Since previous interferometric observations (vB2001 and ONH2004) indicate that Altair's flattening is compatible with uniform rotation, in this paper we adopt the classical Roche model. This model is described in more detail, for example, by Domiciano de Souza et al. (2002), who developed an interferometry-oriented model for rapid rotators. Once the surface equipotential $(\Psi)$ and the corresponding local effective surface gravity $(g(\theta)=|\nabla \Psi|$, where $\theta$ is the colatitude) in the Roche approximation are defined, the local effective temperature is given by the following von Zeipel-like gravity-darkening law (e.g. Collins 1965):

$T_{\text {eff }}(\theta)=T_{\mathrm{p}}\left(\frac{g(\theta)}{g_{\mathrm{p}}}\right)^{\beta}$

where $T_{\mathrm{p}}$ and $g_{\mathrm{p}}$ are the polar effective temperature and gravity, respectively. In this paper we adopt two theoretical limits for the gravity-darkening coefficient $\beta$, namely, $\beta=0.25$ for hot stars with radiative external layers (von Zeipel 1924) and $\beta=$ 0.08 for cold stars with convective external layers (Lucy 1967).

To compute our models of Altair the code BRUCE (Townsend 1997) is used to obtain a stellar grid ( $\simeq 25500$ visible points) for the local values of effective temperature and gravity, velocity field, projected surface, and surface normal direction.

\subsection{Intensity maps}

Because of the geometrical deformation and gravity-darkening, the intensity maps are highly dependent on the inclination of the rotation axis $i$ (Domiciano de Souza et al. 2002). In order to evaluate the intensity maps for Altair we first used Kurucz (1992) model atmospheres ${ }^{2}$ as input for the SYNSPEC code (Hubeny 1988 and Hubeny \& Lanz 1995) to generate a grid of synthetic specific intensities normal to the surface $(I(\mu=1, \lambda)$, where $\mu$ and $\lambda$ have their usual meanings). This grid corresponds to different values of $T_{\text {eff }}$ and $\log g$ in steps of $250 \mathrm{~K}$ and 0.5 dex, respectively. To be consistent with recent spectroscopic observations the grids of $I(\mu=1, \lambda)$ were calculated for microturbulent velocity $v_{\text {micro }}=2 \mathrm{~km} \mathrm{~s}^{-1}$ and solar abundance,

\footnotetext{
2 From Dr. R. L. Kurucz model atmospheres and references publicly available at http://kurucz . harvard.edu/grids /
}

except for 14 elements between $\mathrm{C}$ and $\mathrm{Cu}$ (Erspamer \& North 2002, 2003).

Since interferometric observations of Altair were performed within wide spectral bands, we integrated $I(1, \lambda)$ over the corresponding spectral channel/band to obtain a grid of integrated intensities normal to the surface $I(1)$. Before performing this integration we multiply $I(1, \lambda)$ by the atmospheric and instrumental transmissions. Because the computation of $I(1)$ influences the calculations of interferometric observables, we present further details of this integration procedure in Sect. 3.3.

Once $I(1)$ is defined, we can obtain the intensity at every $\mu(I(\mu))$ through an appropriate limb-darkening law. Accurate modeling of limb-darkening is crucial to determine precise stellar diameters, in particular for rapidly rotating stars where we expect a non-uniform surface brightness distribution. To model Altair we adopted the four-parameter non-linear limbdarkening law proposed by Claret (2000a,b):

$\frac{I(\mu)}{I(1)}=1-a_{1}\left(1-\mu^{\frac{1}{2}}\right)-a_{2}(1-\mu)-a_{3}\left(1-\mu^{\frac{3}{2}}\right)-a_{4}\left(1-\mu^{2}\right)(3)$ where tabulated values of $a_{1}, a_{2}, a_{3}$, and $a_{4}$ are given by Claret for 12 commonly used photometric bands. For Altair we used those for the $H$ and $K$ bands to simulate PTI and VLTI-VINCI observations and those for the $V$ (for $\lambda \leq 600 \mathrm{~nm}$ ) and $R$ (for $\lambda>600 \mathrm{~nm}$ ) bands to simulate NPOI observations.

Claret (2000a,b) argues that Eq. (3) is valid across the whole HR diagram, which is, in fact, an important requirement for a consistent modeling of Altair since this star could present an effective temperature distribution in the transition range between hot (radiative envelope) and cold (convective envelope) stars (e.g., Panzera et al. 1999).

Finally, we can now define the intensity maps $I(\boldsymbol{r})$, i.e., the visible stellar surface brightness distribution on the twodimensional sky-plane at a position $\boldsymbol{r}$. To obtain $I(\boldsymbol{r})$ we thus perform a linear interpolation of the predefined grids of integrated intensities $I(1)$ and the limb-darkening coefficients $a_{1}$, $a_{2}, a_{3}$, and $a_{4}$, for each visible point of the stellar grid.

\subsection{Complex visibilities}

Once the intensity map is defined, the computation of complex visibilities is straightforward. Normalized complex visibilities $V(\boldsymbol{f})$ are obtained by the numerical counterpart of the following equation (for further details see Domiciano de Souza et al. 2002):

$V(\boldsymbol{f})=|V(\boldsymbol{f})| \mathrm{e}^{\mathrm{i} \phi(\boldsymbol{f})}=\frac{\widetilde{I}(\boldsymbol{f})}{\widetilde{I}(\mathbf{0})}$

where $|V|$ and $\phi$ are the visibility amplitude and phase, $\widetilde{I}(\boldsymbol{f})$ is the Fourier transform of the intensity map $I(\boldsymbol{r})$, and $f$ is the spatial frequency coordinate associated with $\boldsymbol{r}$. From Eq. (4) we can directly obtain the interferometric observables: squared visibilities $V^{2}$, triple amplitudes $\left|V_{1}\right|\left|V_{2}\right|\left|V_{3}\right|$, and closure phases $\phi_{1}+\phi_{2}+\phi_{3}$. These observables are functions of the spatial frequency $\boldsymbol{f}$, and the indices 1, 2, and 3 denote the three interferometric baselines in a triangular configuration.

For each pair of telescopes, $\boldsymbol{f}$ is given by the ratio between the vector baseline projected onto the sky $\boldsymbol{B}_{\text {proj }}$ and the 
effective wavelength $\lambda_{\text {eff }}$ of the considered spectral channel: $\boldsymbol{f}=\boldsymbol{B}_{\text {proj }} / \lambda_{\text {efff }}$. This dependence of the spatial frequency on $\lambda_{\text {eff }}$ is responsible for an observational effect known as bandwidth smearing (e.g., Kervella et al. 2003b; and Wittkowski et al. 2004). Indeed, the wide spectral coverage in the $H(\simeq 0.3 \mu \mathrm{m})$ and $K(\simeq 0.4 \mu \mathrm{m})$ bands implies that several spatial frequencies are simultaneously observed by the interferometer (VLTIVINCI and PTI in our case).

To account for the bandwidth smearing in the near-IR we divided the $H$ and $K$ bands into $N=20$ spectral sub-channels and computed the stellar intensity distributions $I_{j}$ integrated over each spectral sub-channel $j$. The corresponding Fourier transforms $\widetilde{I}_{j}$ are then calculated, and the final normalized squared visibilities in the $H$ and $K$ bands are given by:

$V_{\text {IR band }}^{2}=\frac{\sum_{j=1}^{N}\left|\widetilde{I}_{j}\left(\boldsymbol{B}_{\mathrm{proj}} / \lambda_{\mathrm{eff}, j}\right)\right|^{2}}{\sum_{j=1}^{N}\left|\widetilde{I}_{j}(\mathbf{0})\right|^{2}}$.

The bandwidth smearing is negligible for the relatively narrow spectral channels of the NPOI observations.

\section{Results from the $\chi^{2}$ analysis}

In this section we perform a $\chi^{2}$ analysis to constrain a number of unknown physical parameters in our model for Altair from the available interferometric observations.

We investigate two test models corresponding to the theoretical limits for the gravity-darkening parameter $\beta$, namely, 0.08 (convective atmospheres) and 0.25 (radiative atmospheres). To obtain a mean effective temperature compatible with previous works (between $\simeq 7500 \mathrm{~K}$ and $\simeq 8000 \mathrm{~K}$; see for example Esparmer \& North 2003, vB2001; and Ferrero et al. 1995) the adopted polar temperatures $T_{\mathrm{p}}$ for the test models are $8000 \mathrm{~K}$ and $8500 \mathrm{~K}$, corresponding to $\beta=0.08$ and 0.25 , respectively. The chosen stellar mass $M=1.8 M_{\odot}$ is given by Malagnini \& Morossi (1990). Other slightly different mass estimates exist since determining the mass of a single star, particularly a rapid rotator, is not a simple task, but the main results of this work do not critically depend on this value. We adopted the projected equatorial velocity $v_{\mathrm{eq}} \sin i=227 \mathrm{~km} \mathrm{~s}^{-1}$ determined by Reiners \& Royer (2004) from high spectral resolution observations. Their value is compatible with other recent measurements of $v_{\mathrm{eq}} \sin i$ within their error bars (e.g. vB2001; and Royer et al. 2002).

In addition to the fixed physical parameters described above, the equatorial radius $R_{\text {eq }}$ (or the polar one $R_{\mathrm{p}}$ ) is also needed to calculate the models of Altair. However, $R_{\text {eq }}$ is related to the equatorial angular diameter $\oslash_{\mathrm{eq}}$ by means of the stellar distance $\left(d=R_{\mathrm{eq}} / \oslash_{\mathrm{eq}}=5.143 \pm 0.025 \mathrm{pc}\right.$ from Hipparcos; Perryman et al. 1997). Since $\oslash_{\mathrm{eq}}$ is one important output from our interferometric data analysis, $R_{\mathrm{eq}}$ has to be updated accordingly to each $\oslash_{\text {eq }}$ value tested during the $\chi^{2}$ minimization procedure. To avoid calculating a large number of models we performed a preliminary $\chi^{2}$ minimization using a fixed $R_{\text {eq }}$ in order to constraint the range of $\oslash_{\text {eq }}$ close to the

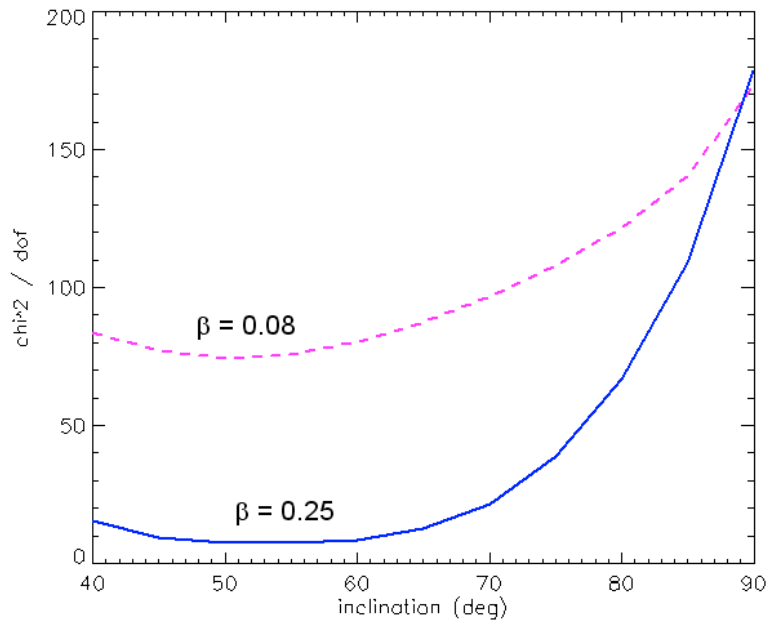

Fig. 1. Reduced $\chi^{2}\left(\chi^{2} /\right.$ d.o.f. $)$ as a function of the stellar inclination $i$ computed from all interferometric observations described in Sect. 2 for the two test models (radiative and convective limits for the gravitydarkening). These values correspond to the best equatorial angular diameter $\oslash_{\text {eq }}$ and major axis orientation $\eta$ for a given $i$. Models with $\beta=0.25$ (solid curve) are preferred compared to models with $\beta=0.08$ (dashed curve). The minimum $\chi^{2} /$ d.o.f. $\left(\chi_{\min }^{2} /\right.$ d.o.f. $\left.=7.3\right)$ is obtained for $\beta=0.25$ and $i=55^{\circ}$. Further physical parameters for this best model from all data (BMAD) and the corresponding error bars are given in Table 3, together with the results of other $\chi^{2}$ analyses.

minimum $\chi^{2}$. In the final $\chi^{2}$ analysis, $\oslash_{\mathrm{eq}}$ (and the corresponding $R_{\mathrm{eq}}$ ) varies with steps of 0.01 mas within a range corresponding to the uncertainty in $\oslash_{\text {eq }}$. Since this uncertainty is rather small $(\$ 2 \%), R_{\mathrm{eq}}$ could be kept constant without introducing any significant changes in our modeling and results.

Additionally, the major axis orientation (position angle) on the sky-plane $\eta$ is allowed to vary in steps of $3^{\circ}$. The inclination of the rotation axis $i$ can vary between $40^{\circ}$ and $90^{\circ}$ (steps of $5^{\circ}$ ). For $i<40^{\circ}$ the equatorial rotation velocity becomes higher than $90 \%$ of the critical limit $\left(v_{\text {crit }}\right)$ leading to unrealistically low equatorial temperatures as a consequence of the von Zeipel effect.

We thus have two test models (corresponding to $\beta=0.08$ and $\beta=0.25)$ with three free parameters $\left(i, \oslash_{\mathrm{eq}}\right.$, and $\left.\eta\right)$ for our $\chi^{2}$ analysis whose results are presented below.

\subsection{Analysis of all data}

We present the results of our $\chi^{2}$ analysis applied to all available interferometric data on Altair (cf. Sect. 2). This consists of 47 near-IR $V^{2}$ observations (VLTI-VINCI and PTI) together with 630 visible observations (NPOI). Figure 1 shows the reduced $\chi^{2}\left(\chi^{2} /\right.$ d.o.f., where the degree of freedom (d.o.f.) is 674) as a function of the inclination $i$ computed from all interferometric observations for the two test models. The values in Fig. 1 correspond to the best $\oslash_{\mathrm{eq}}$ and $\eta$ for a given $i$.

An important result seen in Fig. 1 is that all models with a gravity-darkening coefficient for hot stars $(\beta=0.25)$ are preferred, i.e., have lower $\chi^{2}$ in comparison to models with a gravity-darkening coefficient for cold stars $(\beta=0.08)$. This is model-dependent but still the first direct determination of the gravity-darkening coefficient for a rapid rotator, obtained 

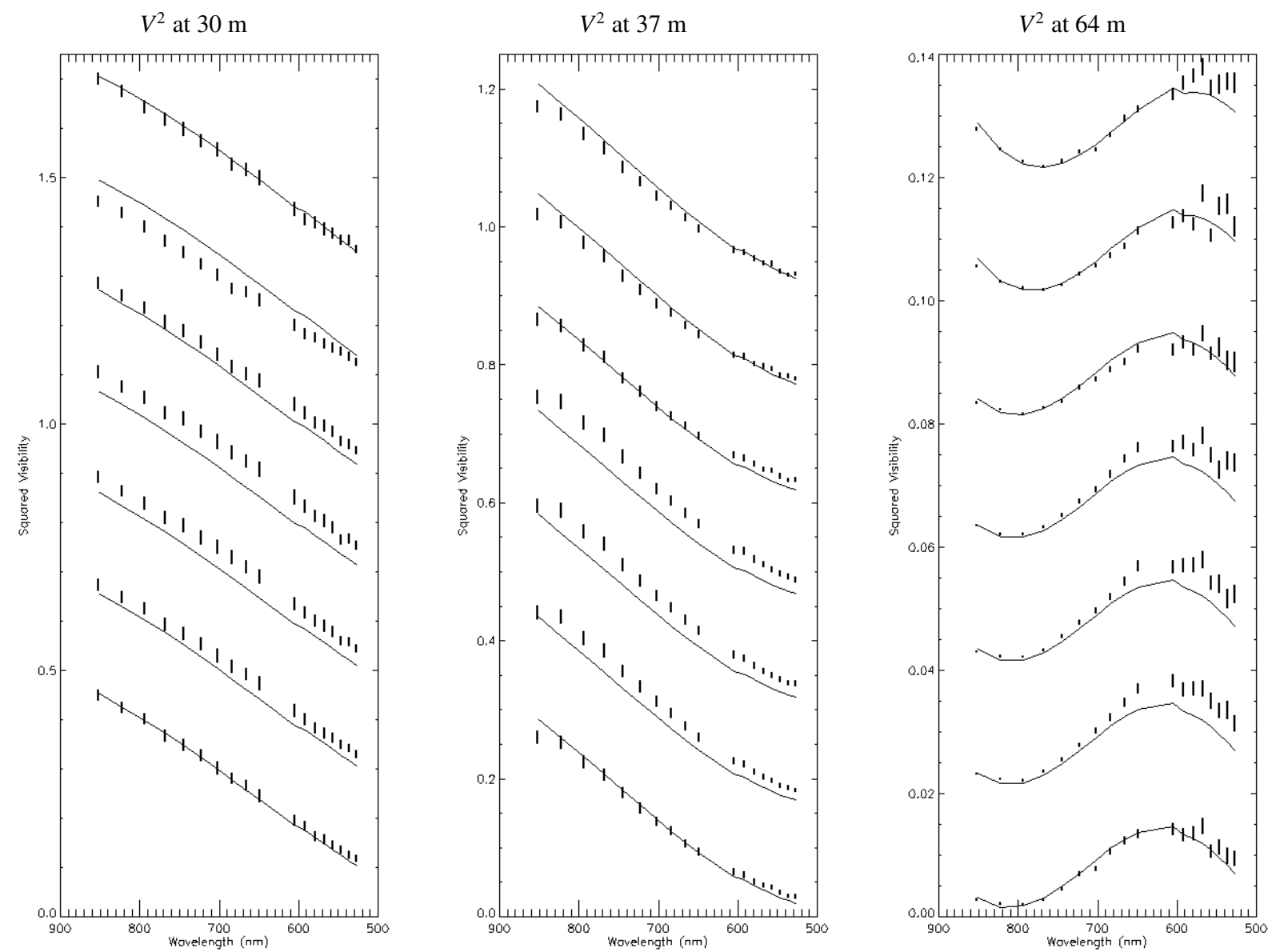

Fig. 2. Squared visibilities $V^{2}$ and corresponding errors versus the wavelength for the seven NPOI scans (see Sect. 2). Solid curves correspond to theoretical values obtained from our best model from the $\chi^{2}$ analysis of all interferometric data (BMAD; see also Fig. 1). Plots for scans 2 to 7 were progressively shifted for better visualization.

thanks to a physically coherent modeling dedicated to stellar interferometry.

The minimum $\chi^{2} /$ d.o.f. is $\chi_{\min }^{2} /$ d.o.f. $=7.3$ obtained for an inclination $i=55^{\circ} \pm 8^{\circ}$. This best model obtained from the $\chi^{2}$ analysis of all data is hereafter refereed as BMAD (best model for all data). All free parameters $\left(\oslash_{\mathrm{eq}}, \eta\right.$, and $i$ ) and uncertainties corresponding to the BMAD are given in Table 3 together with some selected dependent parameters. In Table 3 we also list the results from additional $\chi^{2}$ analyses described in the following sections.

In order to avoid an underestimation of the uncertainties on the free parameters, we computed the limits of the confidence domain by searching for the region between $\chi^{2} /$ d.o.f. and $\chi^{2} /$ d.o.f. +1 . We used the reduced $\chi^{2}$ and not the total $\chi^{2}$ as we found it difficult to account for possible correlations between the error bars on each measurement, in particular for the NPOI data. By adopting $\chi^{2} /$ d.o.f., we chose the conservative approach to consider that all measurements are fully correlated with each other, i.e. that their error bars cannot be diminished by averaging in the fitting process. This means that our derived error bars may be overestimated, but this will avoid an overinterpretation of the data.

In Figs. 2 and 3 we compare the five NPOI observables ( $V^{2}$ for three baselines, triple amplitudes, and closure phases) with the corresponding theoretical values derived from the BMAD. We note in particular that, although the uncertainties in the closure phases are quite small ( $\$ 0.03 \mathrm{rad})$, there is a rather good agreement between the observed closure phases and those obtained from the BMAD (solid curves). Clearly, models with $\beta=0.08$ (plotted as dashed curves for comparison) cannot reproduce these data, leading to $\chi^{2} /$ d.o.f. $>75$ in Fig. 1 . In Fig. 4 we compare the theoretical squared visibilities $V^{2}$ from the BMAD with the observed $V^{2}$ and corresponding errors from VLTI-VINCI ( $H$ and $K$ bands) and PTI ( $K$ band), as described in Sect. 2.

Considering all these distinct interferometers, observables, wavelengths, and baselines (lengths and position angles), Figs. 2 to 4 show a good general agreement between observations and the BMAD, particularly for the closure phases. However, some discrepancies between theoretical and observed $V^{2}$ exist, leading to a relatively a high $\chi_{\min }^{2} /$ d.o.f.(=7.3). This issue is discussed hereafter.

\subsection{Analysis of selected data subsets}

The high $\chi_{\min }^{2} /$ d.o.f. obtained in the last section from the analysis of all interferometric data is partially due to an underestimation of long-term errors for the NPOI visibility amplitudes. 
Triple Amplitude

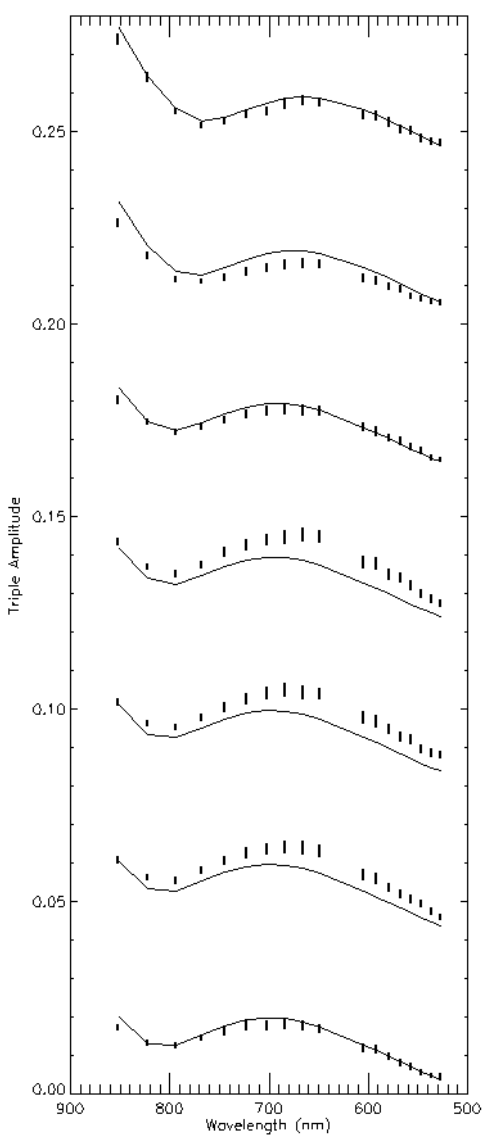

Closure Phase

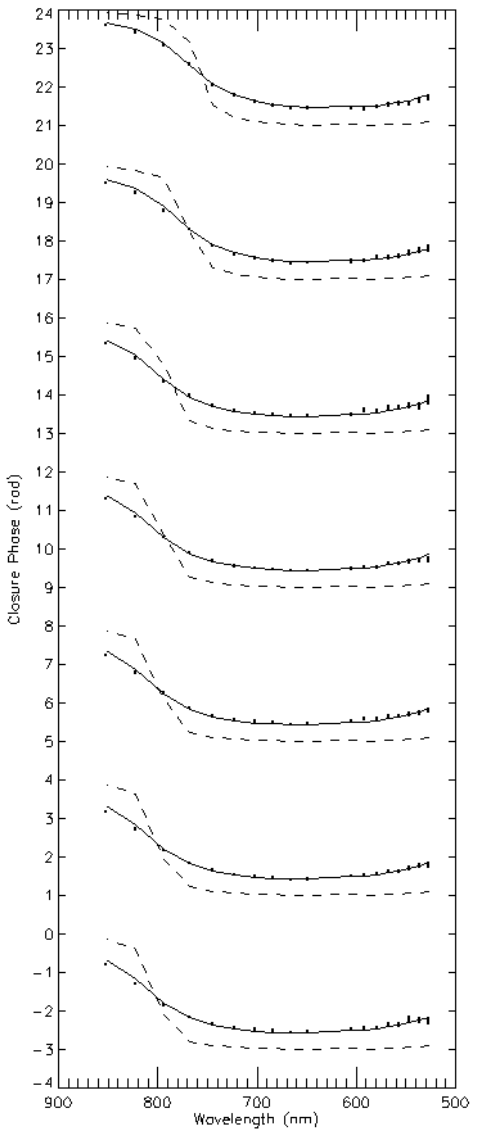

$T_{\text {eff }}$ map (BMAD)

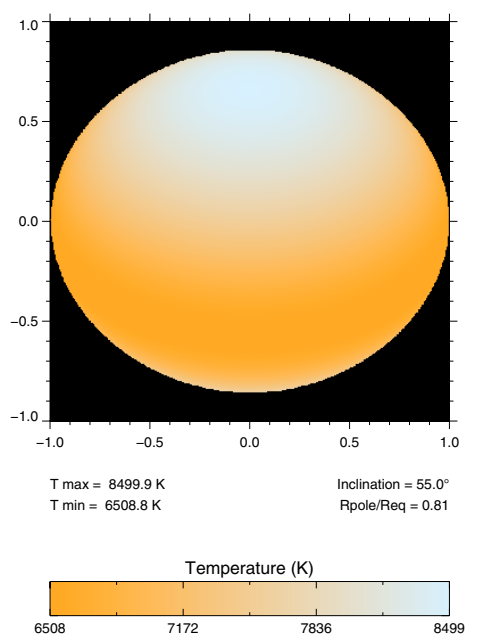

Fig. 3. Triple amplitudes $\left|V_{1}\right|\left|V_{2}\right|\left|V_{3}\right|$, closure phases $\phi_{1}+\phi_{2}+\phi_{3}$, and corresponding errors versus the wavelength for the seven NPOI scans (Sect. 2). Solid curves correspond to theoretical values obtained from our best model from the $\chi^{2}$ analysis of all interferometric data (BMAD; see also Fig. 1). The closure phase is very sensitive to the stellar intensity distribution. Therefore, a comparison between a strong $(\beta=0.25$; solid curves) and a weak ( $\beta=0.08$; dashed curves) gravity-darkened model shows that a highly non-uniform surface brightness distribution is mandatory to reproduce the observed closure phases. Note that the closure phases have small error bars ( $\leqslant 0.03$ rad). Plots for scans 2 to 7 were progressively shifted for better visualization. The picture in the right is the effective temperature map for the BMAD (Table 3 ).

This calibration problem is clearly present in Figs. 2 and 3 as a scatter of the observed $V^{2}$ and triple amplitudes relative to the model. The observations for a given scan are shifted in the same direction for all wavelengths. On the other hand, the closure phase is a more stable interferometric observable, being unaffected by this calibration problem as shown by the excellent agreement between observation and model in Fig. 3 .

We have thus performed another $\chi^{2}$ analysis including only the $(7 \text { scans })^{*}(18$ wavelengths $)$ closure phases from NPOI, together with the 47 near-IR $V^{2}$ from PTI and VINCI. The $\chi^{2} /$ d.o.f. behavior is similar to that seen in Fig. 1, but the minimum reduced $\chi^{2}$ is now $\gtrsim 2$ times smaller than before, namely, $\chi_{\min }^{2} /$ d.o.f. $=3.2$. In agreement with the analysis of all data presented in the last section, we obtained $\beta=0.25$ and $i=55^{\circ} \pm 14^{\circ}$. Further physical parameters for this best model determined from the near-IR $V^{2}$ and closure phases (BMIRCP) are given in Table 3 .

Even though this analysis showed that $\chi_{\min }^{2} /$ d.o.f. $(=3.2)$ diminishes when the NPOI $V^{2}$ and triple amplitudes are removed, the value obtained indicates that some non negligible discrepancies between model and observations still exist. Such discrepancies come from the fact that the near-IR $V^{2}$ for the BMAD and the BMIRCP systematically underestimate the observations from PTI and VINCI, as we can see in Fig. 4.

Because these near-IR $V^{2}$ include data from two distinct interferometers using different calibrators, one can hardly invoke some kind of calibration problem, such as those found on the NPOI data. These low theoretical near-IR $V^{2}$ seem to be due to the rather large equatorial angular diameter deduced from the $\chi^{2}$ minimization, namely, $\oslash_{\mathrm{eq}}=3.83 \pm 0.06$ mas for the BMAD and $\oslash_{\text {eq }}=3.88 \pm 0.08$ mas for the BMIRCP (Table 3). To investigate this point we performed two additional $\chi^{2}$ analyses: one for the ( 7 scans)*(18 wavelengths) closure phases alone (NPOI data) and another for the 47 near-IR $V^{2}$ alone (VLTI-VINCI and PTI data). These results are also summarized in Table 3.

Our analysis result in $\chi_{\min }^{2} /$ d.o.f. $=1.4$ for the best model for the closure phases alone (BMCP). The $\chi^{2} /$ d.o.f. behavior is once more similar to that seen in Fig. 1, resulting in $\beta=0.25$ and $i=50^{\circ} \pm 12^{\circ}$. The derived equatorial diameter $\left(\oslash_{\mathrm{eq}}=\right.$ $3.88 \pm 0.03$ mas) is compatible with those from the two previous analyses (BMAD and BMIRCP). 


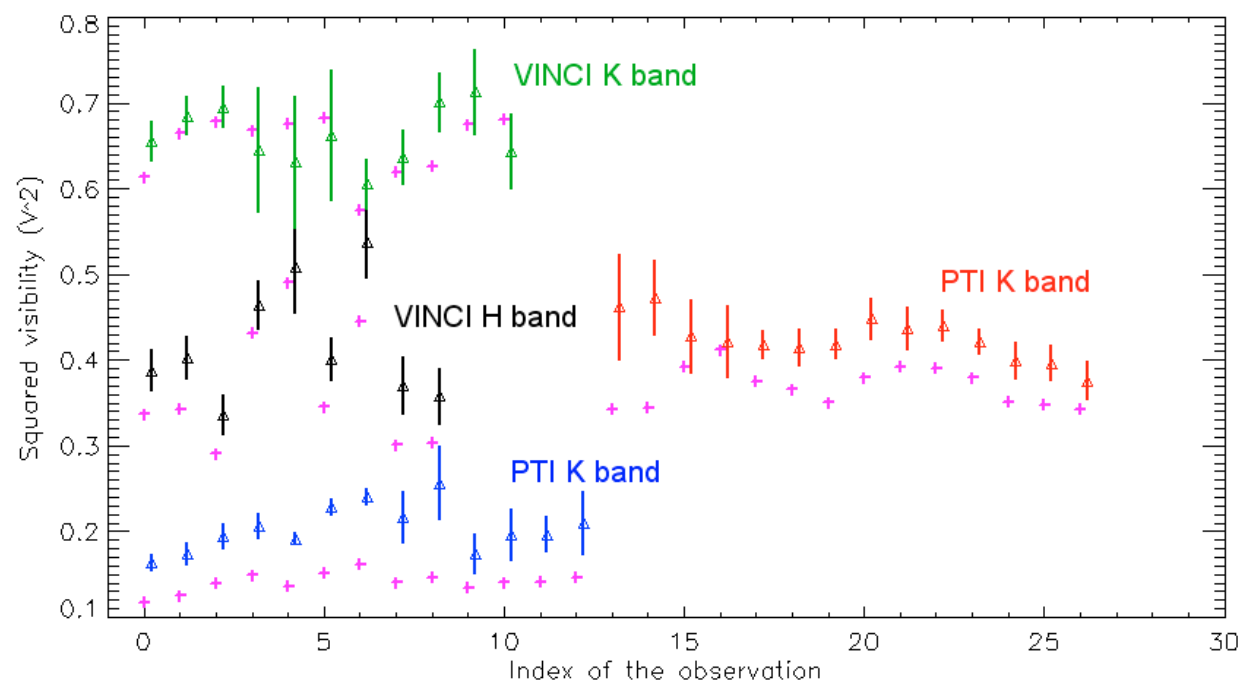

Fig. 4. Squared visibilities $V^{2}$ (triangles) and corresponding error bars from VLTI-VINCI ( $H$ and $K$ bands) and PTI ( $K$ band; baselines $85 \mathrm{~m}$ and $110 \mathrm{~m}$ ) as described in Sect. 2. The abscissa is an index (integer number) to label each group of $V^{2}$ data. The crosses correspond to theoretical $V^{2}$ obtained from the model with minimum $\chi^{2}$ (best model; cf. Fig. 1). The abscissas of the $V^{2}$ data are slightly shifted to the right compared to those of the theoretical $V^{2}$ for better visualization.

Table 3. Input and derived parameters obtained from a $\chi^{2}$ minimization procedure applied to several data sets: all data (BMAD), near-IR $V^{2}$ and closure phases (BMIRCP), closure phases alone (BMCP), and near-IR $V^{2}$ alone (BMIR). There is no uncertainty associated to $\beta$ and $T_{\mathrm{p}}$ because they define two test models based on theoretical limits for the gravity darkening (see text for details). Selected dependent parameters for the best models are also listed.

\begin{tabular}{ccccc}
\hline \hline Fixed input parameters & BMAD & BMIRCP & BMCP & BMIR \\
\hline$v_{\text {eq }} \sin i\left(\mathrm{~km} \mathrm{~s}^{-1}\right)$ & 227 & 227 & 227 & 227 \\
$M\left(M_{\odot}\right)$ & 1.8 & 1.8 & 1.8 & 1.8 \\
$i(\mathrm{deg})$ & - & - & - & $50^{\circ}$ \\
$\left(\beta, T_{\mathrm{p}}(\mathrm{K})\right)$ & - & - & - & $(0.25,8500)$ \\
\hline Results of the $\chi^{2}$ analyses & $\mathrm{BMAD}$ & $\mathrm{BMIRCP}$ & $\mathrm{BMCP}$ & $\mathrm{BMIR}$ \\
\hline$\chi_{\text {min }}^{2} / \mathrm{d} . \mathrm{o} . \mathrm{f}$. & 7.3 & 3.2 & 1.5 & 0.50 \\
$\left(\beta, T_{\mathrm{p}}(\mathrm{K})\right)^{a}$ & $(0.25,8500)$ & $(0.25,8500)$ & $(0.25,8500)$ & - \\
$i(\mathrm{deg})$ & $55^{\circ} \pm 8^{\circ}$ & $55^{\circ} \pm 14^{\circ}$ & $50^{\circ} \pm 12^{\circ}$ & - \\
$2 a=\oslash_{\mathrm{eq}}(\operatorname{mas})$ & $3.83 \pm 0.06$ & $3.88 \pm 0.08$ & $3.88 \pm 0.03$ & $3.44 \pm 0.05$ \\
$R_{\mathrm{eq}}{ }^{\circ}\left(R_{\odot}\right)$ & $2.117 \pm 0.035$ & $2.145 \pm 0.045$ & $2.145 \pm 0.020$ & $1.902 \pm 0.029$ \\
$\eta(\mathrm{deg})$ & $92^{\circ} \pm 6^{\circ}$ & $62^{\circ} \pm 17^{\circ}$ & $95^{\circ} \pm 23^{\circ}$ & $113^{\circ} \pm 12^{\circ}$ \\
\hline Dependent parameters & $\mathrm{BMAD}$ & $\mathrm{BMIRCP}$ & $\mathrm{BMCP}$ & $\mathrm{BMIR}$ \\
\hline$T_{\text {eq }}(\mathrm{K})$ & 6509 & 6483 & 6171 & 6453 \\
$v_{\text {eq }}\left(\mathrm{km} \mathrm{s} \mathrm{s}^{-1}\right)$ & 277 & 277 & 296 & 296 \\
$v_{\text {eq }} / v_{\text {crit }}(\%)$ & $76 \%$ & $76 \%$ & $80 \%$ & $77 \%$ \\
$f_{\text {rot }}(\mathrm{cycles} / \mathrm{day})$ & 2.585 & 2.552 & 2.729 & 3.077 \\
$2 b=\oslash_{\mathrm{p}}^{\max }(\operatorname{mas})$ & 3.29 & 3.33 & 3.32 & 2.99 \\
$a / b=\oslash_{\text {eq }} / \oslash_{\mathrm{p}}^{\max }$ & 1.164 & 1.165 & 1.169 & 1.149 \\
$R_{\text {eq }} / R_{\mathrm{p}}$ & 1.237 & 1.240 & 1.275 & 1.243 \\
\hline
\end{tabular}

${ }^{a}$ Theoretical limit preferred compared to $\left(\beta, T_{\mathrm{p}}(\mathrm{K})\right)=(0.08,8000)$.

${ }^{b}$ From $\oslash_{\text {eq }}$ and Hipparcos distance $(d=5.143 \pm 0.025 \mathrm{pc})$.

Before analyzing the near-IR $V^{2}$ alone, we should note that since VLTI-VINCI and PTI data correspond to observations in the first visibility lobe far from the first minimum and in a limited range of baseline position angles, this analysis suffers from a significant uniqueness problem (Domiciano de Souza et al. 2002). This means, in particular, that the stellar inclination cannot be derived from these data. Thus, we fixed $\beta=0.25$ and $i=50^{\circ}$, compatibly with the values derived for the BMCP. The obtained $\chi_{\min }^{2} /$ d.o.f. is 0.50 for the best model for the near-IR $V^{2}$ alone (BMIR). As expected, the derived equatorial diameter is significantly smaller $\left(\oslash_{\mathrm{eq}}=3.44 \pm 0.05\right.$ mas) than all previous analyses, which included visible data from NPOI.

Figure 5 shows the fit to the closure phase for the BMCP (solid curves in the left panel) and the fit to the near-IR $V^{2}$ 
Closure Phase (visible)

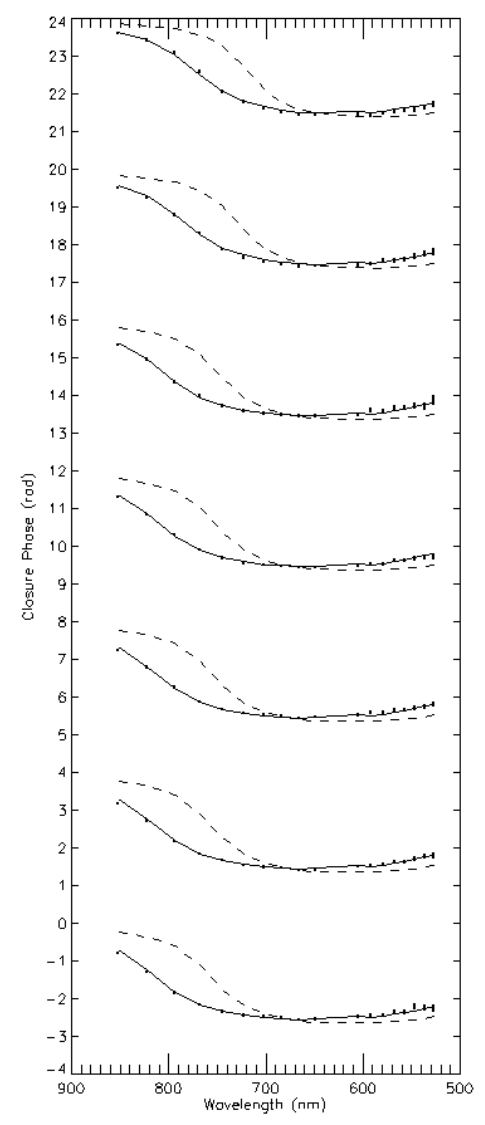

$V^{2}($ near-IR)

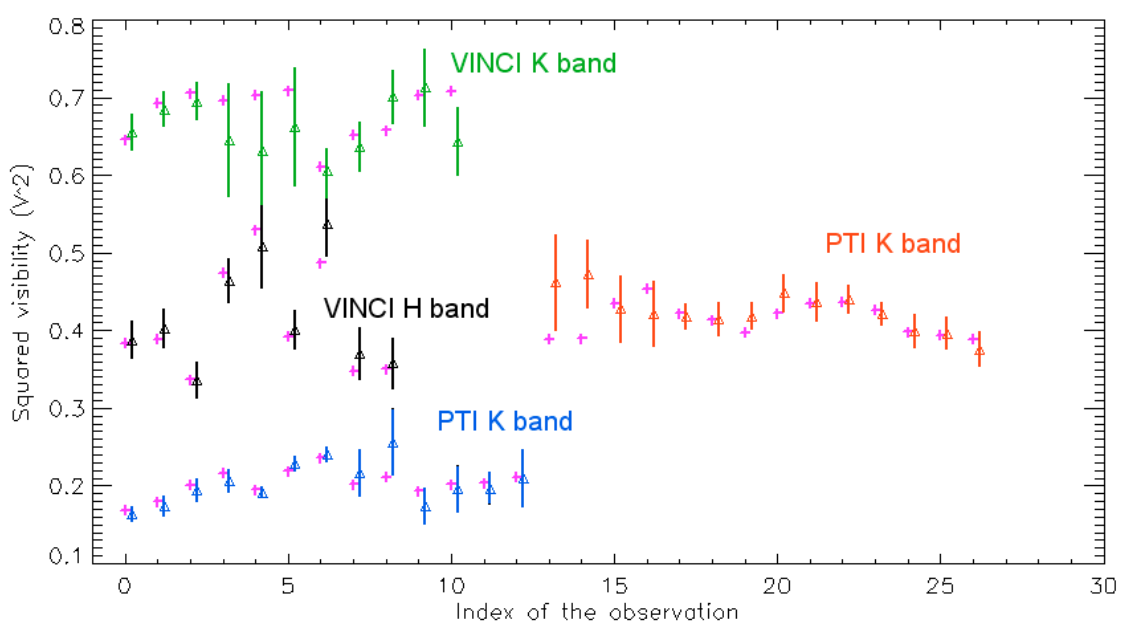

Fig. 5. The left panel shows the closure phases $\phi_{1}+\phi_{2}+\phi_{3}$ and corresponding errors versus the wavelength for the seven NPOI scans (Sect. 2). Solid curves correspond to theoretical values obtained from our best model from the $\chi^{2}$ analysis of closure phases alone (BMCP; see Table 3). Dashed curves correspond to the theoretical closure phases obtained by fixing $i=50^{\circ}, \oslash_{\mathrm{eq}}=3.44$ mas, and $\eta=113^{\circ}$. These parameters were derived from the $\chi^{2}$ analysis of near-IR $V^{2}$ alone (BMIR). The solid curves provide a much better fit to the observed closure phases because they correspond to an angular equatorial diameter larger $\left(\oslash_{\mathrm{eq}}=3.88 \mathrm{mas}\right)$ than the value for the dashed curves $\left(\oslash_{\mathrm{eq}}=3.44\right.$ mas $)$. On the other hand, a model (BMIR) with $\oslash_{\mathrm{eq}}=3.44$ mas nicely fits the near-IR $V^{2}$ as shown in the right panel (see also Fig. 4).

for the BMIR (right panel). Individually, these fits are rather good as one can see in Fig. 5 and also as indicated by the corresponding $\chi_{\min }^{2} /$ d.o.f. (1.4 for the BMCP and 0.5 for the BMIR). However, it is clear that the large $\oslash_{\text {eq }}$ from the analyses including the closure phases cannot fit the near-IR $V^{2}$ (as already shown in Fig. 4).

Conversely, the smaller $\oslash_{\text {eq }}$ derived from the near-IR $V^{2}$ alone (BMIR) cannot fit the closure phases. This is shown as dashed curves in Fig. 5 (left panel), where we plotted the theoretical closure phases obtained by a model with fixed $i=50^{\circ}$, $\beta=0.25, \oslash_{\mathrm{eq}}=3.44 \mathrm{mas}$, and $\eta=113^{\circ}$ (values from the BMIR). Although this model certainly leads to a high $\chi^{2}$, we note that models with $\beta=0.08$ and free $i, \oslash_{\text {eq }}$, and $\eta$, lead to an even higher $\chi^{2}$. Thus, the identification of the von Zeipel effect on Altair $\left(T_{\text {eff }} \propto g^{0.25}\right)$, which is the main result of this work, is not affected or hampered by this discrepancy between the angular sizes derived from the visible and near-IR data.

In the following section we investigate this discrepancy and discuss some physical consequences of our results, in particular concerning the von Zeipel effect.

\section{Discussion}

\subsection{The size of Altair}

The results described in the last section and summarized in Table 3 reveal a discrepancy between the stellar angular diameters required to fit the visible and near-IR interferometric data. This discrepancy also appears when we compare the results obtained in the visible by ONH2004 (NPOI data) and in the $K$ band by vB2001 (PTI data).

To investigate this issue we estimate the angular size of Altair using an independent method: the average surface brightness. Using the surface brightness relations from Kervella et al. (2004b), we can derive the mean equivalent limbdarkened angular diameter of Altair using only its photometric properties (Table 4). We adopted the apparent magnitudes in the visible and near-IR from Hipparcos (Perryman et al. 1997), Morel \& Magnetat (1978), Ducati (2002), and the recent infrared catalogue from Kidger \& Martín-Luis (2003). The error bars from the original authors on the apparent magnitudes are given for each band, except for the $U, R$, and $I$ bands, where 
Table 4. Apparent and absolute magnitudes of Altair, using the Hipparcos parallax ( $\pi=194.45 \pm 0.94$ mas).

\begin{tabular}{lccccccccc}
\hline \hline & $U$ & $B$ & $V$ & $R$ & $I$ & $J$ & $H$ & $K$ & $L$ \\
\hline$m_{\lambda}$ & 1.07 & 0.99 & 0.77 & 0.62 & 0.48 & 0.327 & 0.228 & 0.205 & 0.20 \\
$\sigma\left(m_{\lambda}\right)$ & 0.05 & 0.01 & 0.01 & 0.05 & 0.05 & 0.009 & 0.008 & 0.005 & 0.01 \\
$M_{\lambda}$ & 2.51 & 2.43 & 2.21 & 2.06 & 1.92 & 1.77 & 1.67 & 1.65 & 1.64 \\
\hline
\end{tabular}

a conservative 0.05 mag error has been assumed. No interstellar extinction is taken into account for this nearby $\operatorname{star}(d \simeq 5 \mathrm{pc})$.

We obtain consistent limb-darkened disk angular diameters for all the visible-infrared colors, with, for instance, $\oslash_{\mathrm{LD}}(B, B-$ $L)=3.258 \pm 0.034$ mas. Considering the Hipparcos parallax of $\pi=194.45 \pm 0.94$ mas (distance $d=5.143 \pm 0.025 \mathrm{pc}$ ), this translates into a photometric equivalent linear radius of $1.801 \pm 0.021 R_{\odot}$. Erspamer \& North (2003) obtain an effective temperature of $T_{\text {eff }}=7550 \mathrm{~K}$, averaged over the disk of Altair. The $\oslash_{\mathrm{LD}}\left(T_{\text {eff }}, m_{\lambda}\right)$ relations from Kervella et al. (2004b) give the same LD angular diameter using the $H, K$, and $L$ apparent magnitudes.

Let us compare the photometric-average angular size derived above $\left(\oslash_{\mathrm{LD}}(B, B-L)=3.258 \pm 0.034\right.$ mas $)$ with an equivalent angular disk diameter leading to the same area of the stellar surface projected onto the sky-plane $\bar{\varnothing}$ obtained from our models. For BMIR we obtain $\bar{\emptyset}=3.20$ mas, while for BMAD, BMIRCP, and BMCP we obtain $\bar{\oslash}$ between 3.53 and 3.58 mas. This comparison points towards a smaller size of Altair since there is a better agreement between the sizes estimated by the surface brightness method and by the $\chi^{2}$ analysis including near-IR $V^{2}$ alone, with the latter still being slightly smaller.

From the present data it is not possible to determine whether the discrepancy between the stellar angular diameters in the visible and near-IR has a physical or an instrumental origin. Bias in the wavelength calibration could lead to a larger or smaller size since it affects the spatial frequency. Previous comparisons between stellar angular diameters measured by NPOI and other interferometers show no sign of systematic differences (Nordgren et al. 2001). On the other hand, the large angular size in the visible could also be explained, for example, by an extended emission only seen in the visible. To further investigate the origin of this discrepancy, more precise interferometric observations of Altair are required, preferably in the near-IR at the second visibility lobe and/or with phase closures. These observations should be made in such a way that the quality and the nature of the data in the visible and near-IR are similar and, thus, better comparable. Such observations are expected to be performed with the instrument VLTI-AMBER (e.g., Petrov et al. 2003).

\subsection{Rotation and gravity darkening laws}

In the present paper we consistently adopted the Roche approximation and a von Zeipel-like gravity-darkening (Eq. (2)). Nevertheless, other more subtle possibilities exist and should be considered in the future when more precise interferometric observations of Altair will be available.

For example, our results indicate that the effective temperature at Altair's equator could be low enough that the star presents convection in its external equatorial regions $\left(T_{\mathrm{eq}} \simeq\right.$ $6500 \mathrm{~K}$ for the models with minimum $\chi^{2}$ ). Such low $T_{\text {eq }}$ requires a gravity-darkening exponent $\beta \simeq 0.08$ (Lucy 1967; Claret 2000c), so that a latitudinal dependent $\beta$ parameter should be more convenient for Altair (e.g., a continuous variation from the radiative limit $\beta=0.25$ to the convective limit $\beta=0.08$ between the poles and the equator). The hypothesis of a convective equatorial region is supported by several works showing that Altair has a chromosphere and a corona, possibly linked to subphotospheric convective zones (e.g., Ferrero et al. 1995).

Other possibilities for a variable parameter $\beta$ or even for an alternative gravity-darkening law is the presence of differential rotation (e.g., Connon Smith \& Worley 1974; Kippenhahn 1977). Although Reiners \& Royer (2004) found no signatures of external latitudinal-dependent differential rotation in Altair, we think that one cannot exclude internal differential rotation and/or external differential rotation not detected by the Fourier transform method used by those authors. In fact, the method used by Reiners \& Royer (2004) is not very sensitive to differential rotation laws where polar regions rotate faster than layers closer to the equator (anti solar-like or negative differential rotation; e.g., Reiners \& Schmitt 2002).

Interestingly enough, Stoekley (1968) found evidence of an anti-solar-like differential rotation in Altair. The presence of negative surface differential rotation is in fact compatible with the hypothesis of a convective equatorial region. For such cool regions, characteristic of $\mathrm{F}$ and later type stars, it is possible that some braking mechanism (magnetic effects and/or viscosity due to the onset of convection) could act preferentially close to Altair's equator, slowing these regions relative to those closer to the poles.

This important issue concerning the presence of differential rotation in Altair, and the corresponding gravity-darkening law, should be investigated in the future by additional studies, preferably with distinct and complementary techniques. For example, Domiciano de Souza et al. (2004) proposed a technique to detect both solar-like and anti-solar-like differential rotation by combining high angular resolution with high spectral resolution (differential interferometry). The forthcoming VLTIAMBER will be able to operate in a differential interferometry mode in the near-IR (e.g., Petrov et al. 2003).

\subsection{Inclination and rotation frequency}

The results presented in Sect. 4 suggest an intermediate inclination for Altair (see Table 3). These results seem to disagree with the analysis from Reiners \& Royer (2004), which points towards higher inclinations $\left(i>68^{\circ}\right.$ on a $1 \sigma$ level). However, this discrepancy disappears if we consider $2 \sigma$ confidence 
levels in the results from Reiners \& Royer (2004), which implies $i>45^{\circ}$. Additionally, Stoeckley (1968) derived an inclination angle between $30^{\circ}$ and $50^{\circ}$ from the analysis of line profiles of Mg II 4481 and Ca II 3934, which is in agreement with our results as well.

It is important that further studies investigate how subtle effects, such as differential rotation and gravity-darkening, influence the interferometric observables and, in particular, the line profiles. It would thus be possible to consistently combine interferometry and spectroscopy in order to determine the stellar inclination more precisely.

Once $i, R_{\mathrm{eq}}$, and $v_{\mathrm{eq}} \sin i$ are known, the rotation frequency can be determined, in the uniform rotation approximation, by:

$f_{\text {rot }}=\frac{1}{P_{\text {rot }}}=\frac{\left(v_{\mathrm{eq}} \sin i\right)}{2 \pi R_{\mathrm{eq}}(\sin i)}$.

The rotation frequencies computed from the equation above and corresponding to our $\chi^{2}$ analyses are listed in Table 3 . Recently, Buzasi et al. (2005) detected several pulsation modes in WIRE (Wide-field InfraRed Explorer) observations, indicating that Altair is a low-amplitude $\delta$ Scuti star. The authors suggest that the two low-frequency modes found $\left(f_{1}=\right.$ $2.570 \pm 0.020$ cycles/day and $f_{2}=3.526 \pm 0.020$ cycles/day) may be associated with the stellar rotation frequency.

The theoretical $f_{\text {rot }}$ values listed in Table 3 suggest that the frequency $f_{1}$ measured by Buzasi et al. (2005) is a better candidate for $f_{\text {rot }}$. However, more precise measurements should be acquired before we can unambiguously identify $f_{1}=$ $2.570 \pm 0.020$ cycles/day as the rotation frequency of Altair.

\subsection{On the age of Altair}

This work, along with previous ones, has proven that long baseline interferometry is a powerful technique for studying rapidly rotating stars. In particular, this technique can provide important clues to the many unanswered questions concerning the structure and evolution of rapid rotators. For example, placing an intermediate-mass star like Altair in its evolutionary history is an interesting but difficult task requiring as much information as possible.

The age of a star can be derived using different indicators. Lachaume et al. (1999) have used five different methods to study main-sequence stars in the solar neighborhood: isochrones in the HR diagram, rotation, calcium emission lines, kinematics in the Galaxy, and iron abundance. Unfortunately, due to Altair's rapid rotation, its spectral lines are very broad, and this prevents the application of the $\mathrm{Ca}$ emission line and kinematic methods. The rotational velocity of a hot, fast rotator is not an accurate indicator of its age, because of the uncertainties on the initial rotational velocity and on the braking mechanisms possibly present.

To estimate the age of Altair, we took advantage of the recent models from Girardi et al. (2002). We read the tables of these authors for the following parameters: $[\mathrm{Fe} / \mathrm{H}]=-0.34$, $T_{\text {eff }}=7550 \mathrm{~K}, \log g=4.13$ (Erspamer \& North 2003), and $Z=0.008$. We adopted the absolute magnitudes presented in Table 4, derived using the parallax from Hipparcos
(Perryman et al. 1997): $\pi=194.45 \pm 0.94$ mas. The bolometric magnitude was estimated using the corrections provided by Girardi et al. (2002). The best fit of the models with the observed absolute magnitudes of Altair is obtained for an age between 1.2 and $1.4 \mathrm{Gyr}$.

The relatively old age of Altair suggests that it has kept a high rotation velocity for a long time, well into its lifetime on the main sequence. It does not seem that an efficient braking mechanism is acting to slow down the rotation velocity of this intermediate-mass star. Altair was searched for the presence of a debris disk by Kuchner et al. (1998) in the midinfrared, without success. The absence of a disk could be one of the factors that prevented an efficient slow down of the star by magnetic coupling or turbulent friction. In any case we believe that further studies should be performed on the evolution of an intermediate-mass and fast rotating star such as Altair.

\section{Summary and conclusions}

We performed a physically consistent analysis of all available interferometric data on Altair using our interferometry-oriented model for fast rotators. This model includes Roche approximation, limb-darkening from Claret (2000a,b), and a von Zeipellike gravity-darkening law, as described in Sect. 3 and also by Domiciano de Souza et al. (2002). The rich observational set analyzed here includes new data from VLTI-VINCI ( $V^{2}$ in the $H$ and $K$ bands), as well as published data from PTI ( $V^{2}$ in the $K$ band) and NPOI ( $V^{2}$, triple amplitudes, and closure phases in the visible between $520 \mathrm{~nm}$ and $850 \mathrm{~nm}$ ).

In particular, and as already pointed out by $\mathrm{ONH} 2004$, the presence of gravity-darkening in Altair is revealed by the NPOI observations showing (1) a non-zero $V^{2}$ in the first minimum and (2) a smooth variation of the closure phase between 0 and $\pi$ rad. Thanks to our interferometry-oriented model we were able, for the first time, to provide a physical interpretation of all observations from NPOI, PTI, and VLTI-VINCI combined. In particular, we could show that Altair exhibits a gravitydarkening compatible with the theoretically expected value for hot stars (von Zeipel effect): $T_{\text {eff }} \propto g^{0.25}$.

Moreover, with the parameters and models considered here we were able to show that the observations of Altair are better reproduced by models with an intermediate inclination (between $40^{\circ}$ and $65^{\circ}$ including the error bars).

Our analysis also reveals a possible discrepancy between visible and near-IR angular diameters derived from the data that should be further investigated by visible and near-IR observations of high quality, preferably within the second visibility lobe and/or with closure phases.

Further observations should also be performed to investigate the presence of differential rotation on Altair and the corresponding gravity-darkening laws. Precise interferometric observations in the near-IR, allowing us to study differential rotation in particular (Domiciano de Souza et al. 2004), are soon expected for the VLTI spectro-interferometer AMBER (e.g., Petrov et al. 2003).

Acknowledgements. This work has been supported by a postdoctoral fellowship at the Max-Planck-Institut für Radioastronomie 
(Infrared Interferometry Group). We acknowledge Drs. G. Weigelt and T. Driebe for their invaluable suggestions that allowed us to improve the quality of this work.

\section{References}

Abt, H. A., \& Morrell, N. I. 1995, ApJS, 99, 135

Armstrong, J. T., Mozurkewich, D., Rickard, L. J., et al. 1998, ApJ, 496, 550

Bordé, P., Coudé du Foresto, V., Chagnon, G., \& Perrin, G. 2002, A\&A, 393, 183

Buzasi, D. L., Bruntt, H., Bedding, T. R., et al. 2005, ApJ, 619, 1072

Cayrel de Strobel, G., Soubiran, C., Friel, E. D., Ralite, N., \& Francois, P. 1997, A\&AS, 124, 299

Cayrel de Strobel G., Soubiran C., \& Ralite N. 2001, A\&A, 373, 159

Claret, A. 2000a, A\&A, 363, 1081

Claret, A. 2000b, VizieR Online Data Catalog, 336, 31081

Claret, A. 2000c, A\&A, 359, 289

Cohen, M., Walker, R. G., Carter, B., et al. 1999, AJ, 117, 1864

Colavita, M. M., Wallace, J. K., Hines, B. E., et al. 1999, ApJ, 510, 505

Collins, G. W. 1965, ApJ, 142, 265

Connon Smith, R., \& Worley, R. 1974, MNRAS, 167, 199

Coudé du Foresto, V., Ridgway, S., \& Mariotti, J.-M. 1997, A\&AS, 121,379

Domiciano de Souza, A., Vakili, F., Jankov, S., Janot-Pacheco, E. \& Abe, L. 2002, A\&A, 393, 345

Domiciano de Souza, A., Kervella, P., Jankov, S., et al. 2003, A\&A, 407, L47

Domiciano de Souza, A., Zorec, J., Jankov, S., et al. 2004, A\&A, 418, 781

Ducati, J. R. 2002, NASA Ref. Pub. 1294

Erspamer, D., \& North, P. 2002, VizieR Online Data Catalog, 339, 81121

Erspamer, D., \& North, P. 2003, A\&A, 398, 1121

Ferrero, R. F., Gouttebroze, P., Catalano, S., et al. 1995, ApJ, 439, 1011

Girardi, L., Bertelli, G., Bressan, A., et al. 2002, A\&A, 391, 195

Glebocki, R., Gnacinski, P., \& Stawikowski, A. 2000, Acta Astron., 50,509

Glindemann, A., Algomedo, J., Amestica, R., et al. 2003, Proc. SPIE, 4838,89

Hanbury Brown, R. 1974, in The Intensity Interferometer (London: Taylor \& Francis LTD), 151

Hubeny, I. 1988, Comp. Phys. Comm., 52, 103

Hubeny, I., \& Lanz, T. 1995, ApJ, 439, 875
Hummel, C. A., Mozurkewich, D., Armstrong, J. T., et al. 1998, AJ, 116, 2536

Kervella, P., Coudé du Foresto, V., Glindemann, A., \& Hofmann, R. 2000, SPIE, 4006, 31

Kervella, P., Gitton, Ph., Ségransan, D., et al. 2003a, SPIE, 4838, 858

Kervella, P., Thévenin, F., Morel, P., Bordé, P., \& Di Folco, E. 2003b, A\&A, 408, 681

Kervella, P., Ségransan, D., \& Coudé du Foresto, V. 2004a, A\&A, 425, 1171

Kervella, P., Thévenin, F., Di Folco, E., \& Ségransan, D. 2004b, A\&A, 426, 297

Kidger, M. R., \& Martín-Luis, F. 2003, AJ, 125, 3311

Kippenhahn, R. 1977, A\&A, 58, 267

Kuchner, M. J., Brown, M. E., \& Koresko, C. D. 1998, PASP, 110, 1336

Kurucz, R. L. 1992, The Stellar Populations of Galaxies, IAU Symp. 149, 225

Lachaume, R., Dominik, C., Lanz, T., \& Habing, H. J. 1999, A\&A, 348,897

Lebouquin, J. B., Rousselet-Perraut, K., Kern, P., et al. 2004, A\&A, 424, 719

Lucy, L. B. 1967, Z. Astrophys., 65, 89

Malagnini, M. L., \& Morossi, C. 1990, A\&AS, 85, 1015

Morel, M., \& Magnetat, P. 1978, A\&AS, 34, 477

Nordgren, T. E., Sudol, J. J., \& Mozurkewich, D. 2001, AJ, 122, 2707

Ohishi, N., Nordgren, T. E., \& Hutter, D. J. 2004, ApJ, 612, 463

Panzera, M. R., Tagliaferri, G., Pasinetti, L., \& Antonello, E. 1999, A\&A, 348, 161

Perryman, M. A. C., Lindegren, L., Kovalevsky, J., et al., The Hipparcos Catalogue, 1997, A\&A, 323, 49

Petrov, R. G., \& Amber Consortium, The 2003, EAS Publications Series, Observing with the VLTI, ed. G. Perrin and F. Malbet, 6, 111

Reiners, A., \& Schmitt, J. H. M. M. 2002, A\&A, 384, 155

Reiners, A., \& Royer, F. 2004, A\&A, 428, 199

Royer, F., Grenier, S., Baylac, M.-O., Gómez, A. E., \& Zorec, J. 2002, A\&A, 393, 897

Ségransan, D., Forveille, T., Millan-Gabet, C. P. R., \& Traub, W. A. 1999, in ASP Conf. Ser. 194, 290

Stoeckley, T. R. 1968, MNRAS, 140, 121

Townsend, R. H. D. 1997, MNRAS, 284, 839

van Belle, G. T., Ciardi, D. R., Thompson, R. R., Akeson, R. L., \& Lada, E. A. 2001, ApJ, 559, 1155

von Zeipel, H. 1924, MNRAS, 84, 665

Wittkowski, M., Aufdenberg, J. P., \& Kervella, P. 2004, A\&A, 413, 711 